\title{
New Iterative Method: An Application for Solving Fractional Physical Differential Equations
}

\author{
A. A. Hemeda \\ Department of Mathematics, Faculty of Science, Tanta University, Tanta 31527, Egypt \\ Correspondence should be addressed to A. A. Hemeda; aahemeda@yahoo.com \\ Received 12 September 2012; Accepted 7 March 2013 \\ Academic Editor: Soon Y. Chung
}

Copyright (C) 2013 A. A. Hemeda. This is an open access article distributed under the Creative Commons Attribution License, which permits unrestricted use, distribution, and reproduction in any medium, provided the original work is properly cited.

\begin{abstract}
The new iterative method with a powerful algorithm is developed for the solution of linear and nonlinear ordinary and partial differential equations of fractional order as well. The analysis is accompanied by numerical examples where this method, in solving them, is used without linearization or small perturbation which confirm the power, accuracy, and simplicity of the given method compared with some of the other methods.
\end{abstract}

\section{Introduction}

Considerable attention has been devoted to the study of the fractional calculus during the past three decades and its numerous applications in the area of physics and engineering. The applications of fractional calculus used in many fields such as electrical networks, control theory of dynamical systems, probability and statistics, electrochemistry, chemical physics, optics, and signal processing can be successfully modelled by linear or nonlinear fractional differential equations.

So far there have been several fundamental works on the fractional derivative and fractional differential equations [13]. These works are to be considered as an introduction to the theory of fractional derivative and fractional differential equations and provide a systematic understanding of the fractional calculus such as the existence and uniqueness $[4,5]$. Recently, many other researchers have paid attention to existence result of solution of the initial value problem and boundary problem for fractional differential equations [4-6].

Finding approximate or exact solutions of fractional differential equations is an important task. Except for a limited number of these equations, we have difficulty in finding their analytical solutions. Therefore, there have been attempts to develop new methods for obtaining analytical solutions which reasonably approximate the exact solutions. Several such techniques have drawn special attention, such as Adomain's decomposition method [7], homotopy perturbation method [8-10], homotopy analysis method [11, 12], variational iteration method [13-17], Chebyshev spectral method $[18,19]$, and new iterative method [20-22]. Among them, the new iterative method provides an effective procedure for explicit and numerical solutions of a wide and general class of differential systems representing real physical problems. The new iterative method is more superior than the other nonlinear methods, such as the perturbation methods where this method does not depend on small parameters, such that it can find wide application in nonlinear problems without linearization or small perturbation.

The motivation of this paper is to extend the application of the new iterative method proposed by Daftardar-Gejji and Jafari [20-22] to solve linear and nonlinear ordinary and partial differential equations of fractional order. This motivation is based on the importance of these equations and their applications in various subjects in physical branches $[10,11,14,23-25]$.

There are several definitions of a fractional derivative of order $\alpha>0[3,26]$. The two most commonly used definitions are Riemann-Liouville and Caputo. Each definition uses Riemann-Liouville fractional integration and derivative of whole order. The difference between the two definitions 
is in the order of evaluation. Riemann-Liouville fractional integration of order $\alpha$ is defined as

$$
I^{\alpha} f(x)=\frac{1}{\Gamma(\alpha)} \int_{0}^{x}(x-\xi)^{\alpha-1} f(\xi) d \xi, \quad \alpha>0, x>0 .
$$

The next two equations define Riemann-Liouville and Caputo fractional derivatives of order $\alpha$, respectively, as

$$
\begin{aligned}
D^{\alpha} f(x) & =\frac{d^{m}}{d x^{m}}\left(I^{m-\alpha} f(x)\right), \\
D_{*}^{\alpha} f(x) & =I^{m-\alpha}\left(\frac{d^{m}}{d x^{m}} f(x)\right),
\end{aligned}
$$

where $m-1<\alpha<m, m \in N$.

Caputo fractional derivative first computes an ordinary derivative followed by a fractional integral to achieve the desired order of fractional derivative. Riemann-Liouville fractional derivative is computed in the reverse order. Therefore, Caputo fractional derivative allows traditional initial and boundary conditions to be included in the formulation of the problem.

From properties of $D_{*}^{\alpha}$ and $I^{\alpha}$, it is important to note that

$$
D_{*}^{\alpha} x^{\beta}=\frac{\Gamma(\beta+1) x^{\beta-\alpha}}{\Gamma(\beta+1-\alpha)}, \quad \beta \geq \alpha,
$$

where $D_{*}^{\alpha}$ is Caputo derivative operator of order $\alpha$,

$$
I^{\alpha} x^{\beta}=\frac{\Gamma(\beta+1) x^{\beta+\alpha}}{\Gamma(\beta+1+\alpha)} .
$$

\section{Basic Idea of New Iterative Method}

For the basic idea of the new iterative method, we consider the following general functional equation [20-22]:

$$
u(x)=f(x)+N(u(x))
$$

where $N$ is a nonlinear operator from a Banach space $B \rightarrow$ $B$ and $f$ is a known function. We have been looking for a solution of (4) having the series form

$$
u(x)=\sum_{i=0}^{\infty} u_{i}(x)
$$

The nonlinear operator $N$ can be decomposed as

$$
\begin{aligned}
N\left(\sum_{i=0}^{\infty} u_{i}\right)= & N\left(u_{0}\right) \\
& +\sum_{i=1}^{\infty}\left\{N\left(\sum_{j=0}^{i} u_{j}\right)-N\left(\sum_{j=0}^{i-1} u_{j}\right)\right\} .
\end{aligned}
$$

From (5) and (6), (4) is equivalent to

$$
\begin{aligned}
\sum_{i=0}^{\infty} u_{i}= & +N\left(u_{0}\right) \\
& +\sum_{i=1}^{\infty}\left\{N\left(\sum_{j=0}^{i} u_{j}\right)-N\left(\sum_{j=0}^{i-1} u_{j}\right)\right\} .
\end{aligned}
$$

We define the following recurrence relation:

$$
\begin{gathered}
u_{0}=f, \\
u_{1}=N\left(u_{0}\right), \\
u_{n+1}=N\left(u_{0}+u_{1}+\cdots+u_{n}\right) \\
-N\left(u_{0}+u_{1}+\cdots+u_{n-1}\right), \quad n=1,2, \ldots
\end{gathered}
$$

Then,

$$
\begin{gathered}
\left(u_{1}+u_{2}+\cdots+u_{n+1}\right) \\
=N\left(u_{0}+u_{1}+\cdots+u_{n}\right), \quad n=1,2, \ldots, \\
u=\sum_{i=0}^{\infty} u_{i}=f+N\left(\sum_{i=0}^{\infty} u_{i}\right) .
\end{gathered}
$$

If $\|N(x)-N(y)\|<k\|x-y\|, 0<k<1$, then

$$
\begin{aligned}
\left\|u_{n+1}\right\| & \\
& =\left\|N\left(u_{0}+\cdots+u_{n}\right)-N\left(u_{0}+\cdots+u_{n-1}\right)\right\| \\
& \leq k\left\|u_{n}\right\| \leq \cdots \leq k^{n+1}\left\|u_{0}\right\|, \quad n=0,1,2, \ldots,
\end{aligned}
$$

and the series $\sum_{i=0}^{\infty} u_{i}$ absolutely and uniformly converges to a solution of (4) [27], which is unique, in view of the Banach fixed point theorem [28]. The $n$-term approximate solution of (4) and (5) is given by $u(x)=\sum_{i=0}^{n-1} u_{i}$.

2.1. Convergence of the Method. Now we analyze the convergence of the new iterative method for solving any general functional equation (4). Let $e=u^{*}-u$, where $u^{*}$ is the exact solution, $u$ is the approximate solution, and $e$ is the error in the solution of (4); obviously e satisfies (4), that is,

$$
e(x)=f(x)+N(e(x))
$$

and the recurrence relation (8) becomes

$$
\begin{gathered}
e_{0}=f, \\
e_{1}=N\left(e_{0}\right), \\
e_{n+1}=N\left(e_{0}+e_{1}+\cdots+e_{n}\right) \\
-N\left(e_{0}+e_{1}+\cdots+e_{n-1}\right), \quad n=1,2, \ldots .
\end{gathered}
$$




$$
\begin{aligned}
& \text { If }\|N(x)-N(y)\| \leq k\|x-y\|, 0<k<1 \text {, then } \\
& e_{0}=f \text {, } \\
& \left\|e_{1}\right\|=\left\|N\left(e_{0}\right)\right\| \leq k\left\|e_{0}\right\| \text {, } \\
& \left\|e_{2}\right\|=\left\|N\left(e_{0}+e_{1}\right)-N\left(e_{0}\right)\right\| \\
& \leq k\left\|e_{1}\right\| \leq k^{2}\left\|e_{0}\right\|, \\
& \left\|e_{3}\right\|=\left\|N\left(e_{0}+e_{1}+e_{2}\right)-N\left(e_{0}+e_{1}\right)\right\| \\
& \leq k\left\|e_{2}\right\| \leq k^{3}\left\|e_{0}\right\|, \\
& \left\|e_{n+1}\right\|=\left\|N\left(e_{0}+\cdots+e_{n}\right)-N\left(e_{0}+\cdots+e_{n-1}\right)\right\| \\
& \leq k\left\|e_{n}\right\| \leq k^{n+1}\left\|e_{0}\right\|, \quad n=0,1,2, \ldots .
\end{aligned}
$$

Thus $e_{n+1} \rightarrow 0$ as $n \rightarrow \infty$, which proves the convergence of the new iterative method for solving the general functional equation (4). For more details, you can see [29].

\section{Suitable Algorithm}

In this section, we introduce a suitable algorithm for solving nonlinear partial differential equations using the new iterative method. Consider the following nonlinear partial differential equation of arbitrary order:

$$
\begin{gathered}
D_{* t}^{\alpha} u(x, t)=A(u, \partial u)+B(x, t), \\
m-1<\alpha<m, m \in N, \\
\frac{\partial^{k}}{\partial t^{k}} u(x, 0)=h_{k}(x), \quad k=0,1, \ldots, m-1,
\end{gathered}
$$

where $A$ is a nonlinear function of $u$ and $\partial u$ (partial derivatives of $u$ with respect to $x$ and $t$ ) and $B$ is the source function. In view of the new iterative method, the initial value problem (14a) and (14b) is equivalent to the integral equation

$$
u(x, t)=\sum_{k=0}^{m-1} h_{k}(x) \frac{t^{k}}{k !}+I_{t}^{\alpha} B+I_{t}^{\alpha} A=f+N(u),
$$

where

$$
\begin{gathered}
f=\sum_{k=0}^{m-1} h_{k}(x) \frac{t^{k}}{k !}+I_{t}^{\alpha} B, \\
N(u)=I_{t}^{\alpha} A .
\end{gathered}
$$

Remark 1. When the general functional equation (4) is linear, the recurrence relation (8) can be simplified in the form

$$
\begin{gathered}
u_{0}=f, \\
u_{n+1}=N\left(u_{n}\right), \quad n=0,1,2, \ldots .
\end{gathered}
$$

Proof. From the properties of integration and by using (8) and (16b), we have

$$
\begin{aligned}
u_{n+1}= & N\left(u_{0}+\cdots+u_{n-1}+u_{n}\right) \\
& -N\left(u_{0}+\cdots+u_{n-1}\right) \\
= & I_{t}^{\alpha}\left[u_{0}+\cdots+u_{n-1}+u_{n}\right] \\
& -I_{t}^{\alpha}\left[u_{0}+\cdots+u_{n-1}\right] \\
= & I_{t}^{\alpha}\left[u_{0}\right]+\cdots+I_{t}^{\alpha}\left[u_{n-1}\right] \\
& +I_{t}^{\alpha}\left[u_{n}\right]-I_{t}^{\alpha}\left[u_{0}\right]-I_{t}^{\alpha}\left[u_{n-1}\right] \\
= & I_{t}^{\alpha}\left[u_{n}\right]=N\left(u_{n}\right), \quad n=0,1,2, \ldots
\end{aligned}
$$

Therefore, we get the solution of (15) by employing the recurrence relation (8) or (17).

\section{Applications}

To illustrate the effectiveness of the proposed method, several test examples are carried out in this section.

Example 2. In this example, we consider the following initial value problem in the case of the inhomogeneous BagelyTorvik equation $[23,24]$ :

$$
\begin{gathered}
D_{* x}^{2} u(x)+D_{* x}^{1.5} u(x)+u(x)=g(x), \\
u(0)=1, \quad u^{\prime}(0)=1, \quad x \in[0, L],
\end{gathered}
$$

where $g(x)=1+x$. The exact solution of this problem is $u(x)=1+x$.

By applying the technique described in Sections 2 and 3 , the initial value problem (19) is equivalent to the integral equation

$$
u(x)=1+x+\frac{x^{2}}{2}+\frac{x^{3}}{6}-I_{x}^{2}\left[D_{* x}^{1.5} u(x)+u(x)\right] .
$$

Let $N(u)=-I_{x}^{2}\left[D_{* x}^{1.5} u(x)+u(x)\right]$. In view of recurrence relation (17), we have the following first approximations:

$$
\begin{gathered}
u_{0}(x)=1+x+\frac{x^{2}}{2}+\frac{x^{3}}{6}, \\
u_{1}(x)=N\left(u_{0}\right)=-\frac{8 x^{2.5}}{15 \sqrt{\pi}}-\frac{16 x^{3.5}}{105 \sqrt{\pi}} \\
\quad-\frac{x^{2}}{2}-\frac{x^{3}}{6}-\frac{x^{4}}{24}-\frac{x^{5}}{120}, \\
u_{2}(x)=N\left(u_{1}\right)=\frac{8 x^{2.5}}{15 \sqrt{\pi}}+\frac{16 x^{3.5}}{105 \sqrt{\pi}} \\
+\frac{64 x^{4.5}}{945 \sqrt{\pi}}+\frac{128 x^{5.5}}{10395 \sqrt{\pi}}+\frac{x^{3}}{6}+\frac{x^{4}}{12} \\
+\frac{x^{5}}{120}+\frac{x^{6}}{720}+\frac{x^{7}}{5040},
\end{gathered}
$$




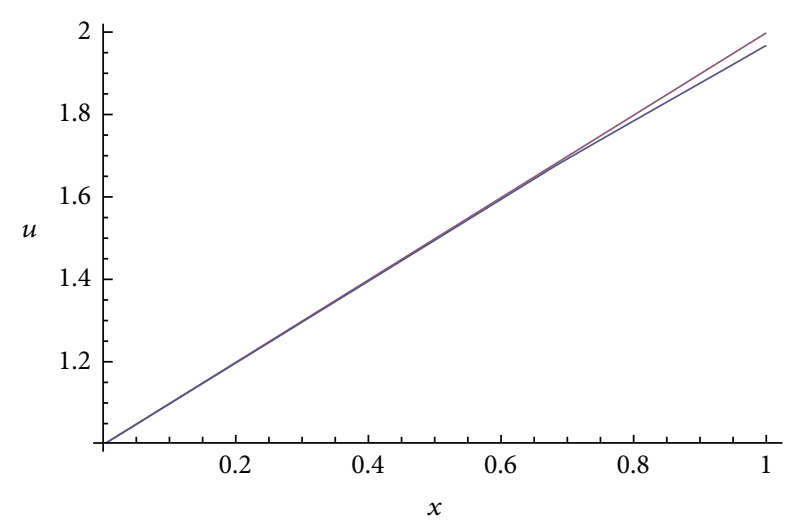

FIGURE 1: Plots of the approximate solution and the exact solution for (19).

and so on. In the same manner the rest of components can be obtained. The 6-term approximate solution for (19) is

$$
\begin{aligned}
u(x)= & \sum_{i=0}^{5} u_{i} \\
= & 1+x-\frac{x^{6}}{144}-\frac{5 x^{7}}{5040}-\frac{x^{9}}{36288} \\
& -\frac{x^{10}}{362880}-\frac{x^{12}}{479001600}-\frac{x^{13}}{6227020800} \\
& -\frac{32 x^{4.5}}{945 \sqrt{\pi}}-\frac{64 x^{5.5}}{10395 \sqrt{\pi}}-\frac{512 x^{7.5}}{405405 \sqrt{\pi}} \\
& -\frac{1024 x^{8.5}}{6891885 \sqrt{\pi}}-\frac{512 x^{10.5}}{687465529 \sqrt{\pi}}-\frac{128 x^{11.5}}{1976463395 \sqrt{\pi}} .
\end{aligned}
$$

Remark 3. In Example 2. we have used the recurrence relation (17). If we used the recurrence relation (8) in place of (17), we obtain the same result.

In Figure 1, we have plotted the 6-term approximate solution with the corresponding exact solution for (19). It is remarkable to note that the two solutions are almost equal.

Comparing these obtained results with those obtained by new Jacobi operational matrix in $[23,24]$, we can confirm the simplicity and accuracy of the given method.

Example 4. Consider the following fractional Riccati equation [10]:

$$
D_{* x}^{\alpha} u(x)+u^{2}(x)=1, \quad u(0)=0, x>0,0<\alpha \leq 1 .
$$

The exact solution when $\alpha=1$ is $u(x)=\left(e^{2 x}-1\right) /\left(e^{2 x}+1\right)$.
By applying the technique described in Sections 2 and 3 , the initial value problem (23) is equivalent to the integral equation

$$
u(x)=\frac{x^{\alpha}}{\Gamma(1+\alpha)}-I_{x}^{\alpha}\left[u^{2}(x)\right]
$$

Let $N(u)=-I_{x}^{\alpha}\left[u^{2}(x)\right]$. In view of recurrence relation (8), we have the following first approximations:

$$
\begin{gathered}
u_{0}(x)=\frac{x^{\alpha}}{\Gamma(1+\alpha)}, \\
u_{1}(x)=N\left(u_{0}\right)=-\frac{\Gamma(1+2 \alpha) x^{3 \alpha}}{\Gamma(1+3 \alpha) \Gamma(1+\alpha)^{2}}, \\
u_{2}(x)=N\left(u_{0}+u_{1}\right)-N\left(u_{0}\right) \\
=\frac{2 \Gamma(1+2 \alpha) \Gamma(1+4 \alpha) x^{5 \alpha}}{\Gamma(1+3 \alpha) \Gamma(1+5 \alpha) \Gamma(1+\alpha)^{3}} \\
-\frac{\Gamma(1+2 \alpha)^{2} \Gamma(1+6 \alpha) x^{7 \alpha}}{\Gamma(1+3 \alpha)^{2} \Gamma(1+7 \alpha) \Gamma(1+\alpha)^{4}},
\end{gathered}
$$

and so on. The 4-term approximate solution for (23) is

$$
\begin{aligned}
& u(x)=\sum_{i=0}^{3} u_{i} \\
& =\frac{x^{\alpha}}{\Gamma(1+\alpha)} \\
& -\frac{\Gamma(1+2 \alpha) x^{3 \alpha}}{\Gamma(1+3 \alpha) \Gamma(1+\alpha)^{2}} \\
& +\frac{2 \Gamma(1+2 \alpha) \Gamma(1+4 \alpha) x^{5 \alpha}}{\Gamma(1+3 \alpha) \Gamma(1+5 \alpha) \Gamma(1+\alpha)^{3}} \\
& -\frac{\Gamma(1+2 \alpha)^{2} \Gamma(1+6 \alpha) x^{7 \alpha}}{\Gamma(1+3 \alpha)^{2} \Gamma(1+7 \alpha) \Gamma(1+\alpha)^{4}} \\
& -\frac{4 \Gamma(1+2 \alpha) \Gamma(1+4 \alpha) \Gamma(1+6 \alpha) x^{7 \alpha}}{\Gamma(1+3 \alpha) \Gamma(1+5 \alpha) \Gamma(1+7 \alpha) \Gamma(1+\alpha)^{4}} \\
& +\frac{4 \Gamma(1+2 \alpha)^{2} \Gamma(1+4 \alpha)}{\Gamma(1+3 \alpha)^{2} \Gamma(1+5 \alpha)} \\
& \frac{\Gamma(1+8 \alpha) x^{9 \alpha}}{\Gamma(1+9 \alpha) \Gamma(1+\alpha)^{5}} \\
& +\frac{2 \Gamma(1+2 \alpha)^{2} \Gamma(1+6 \alpha) \Gamma(1+8 \alpha) x^{9 \alpha}}{\Gamma(1+3 \alpha)^{2} \Gamma(1+7 \alpha) \Gamma(1+9 \alpha) \Gamma(1+\alpha)^{5}} \\
& -\frac{4 \Gamma(1+2 \alpha)^{2} \Gamma(1+4 \alpha)^{2} \Gamma(1+10 \alpha) x^{11 \alpha}}{\Gamma(1+3 \alpha)^{2} \Gamma(1+5 \alpha)^{2} \Gamma(1+11 \alpha) \Gamma(1+\alpha)^{6}} \\
& -\frac{2 \Gamma(1+2 \alpha)^{3}}{\Gamma(1+3 \alpha)^{3} \Gamma(1+7 \alpha)} \\
& \frac{\Gamma(1+6 \alpha) \Gamma(1+10 \alpha) x^{11 \alpha}}{\Gamma(1+11 \alpha) \Gamma(1+\alpha)^{6}}
\end{aligned}
$$




$$
\begin{aligned}
& +\frac{4 \Gamma(1+2 \alpha)^{3} \Gamma(1+4 \alpha) \Gamma(1+6 \alpha)}{\Gamma(1+3 \alpha)^{3} \Gamma(1+5 \alpha) \Gamma(1+7 \alpha)} \\
& \cdot \frac{\Gamma(1+12 \alpha) x^{13 \alpha}}{\Gamma(1+13 \alpha) \Gamma(1+\alpha)^{7}} \\
& -\frac{\Gamma(1+2 \alpha)^{4} \Gamma(1+6 \alpha)^{2} \Gamma(1+14 \alpha) x^{15 \alpha}}{\Gamma(1+3 \alpha)^{4} \Gamma(1+7 \alpha)^{2} \Gamma(1+15 \alpha) \Gamma(1+\alpha)^{8}} .
\end{aligned}
$$

In Figure 2, we have plotted the 4-term approximate solution for (23) for different values of $\alpha$ with the corresponding exact solution. It is remarkable to note that the approximate solution, in case $\alpha=1$, and the exact solution are almost equal (continuous curve) whenever the approximate solution, in cases $\alpha=0.9,0.8$, is of high agreement with the exact solution (dashed and dotted curves, resp.).

Comparing the obtained results with those obtained by homotopy analysis method, in case $h=-1$, in [10], we can confirm the simplicity and accuracy of the given method.

Example 5. Consider the following initial value problem with fractional order $[23,24]$ :

$$
\begin{aligned}
& D_{* x}^{3} u(x)+D_{* x}^{2.5} u(x)+u^{2}(x)=x^{4}, \\
& u(0)=u^{\prime}(0)=0, \quad u^{\prime \prime}(0)=2 .
\end{aligned}
$$

The exact solution for this problem is $u(x)=x^{2}$.

As in Example 4, the initial value problem (27) is equivalent to the integral equation

$$
u(x)=x^{2}+\frac{x^{7}}{210}-I_{x}^{3}\left[D_{* x}^{2.5} u(x)+u^{2}(x)\right] .
$$

Let $N(u)=-I_{x}^{3}\left[D_{* x}^{2.5} u(x)+u^{2}(x)\right]$. In view of recurrence relation (8), we have the following first approximations:

$$
\begin{aligned}
& u_{0}(x)=x^{2}+\frac{x^{7}}{210}, \\
& u_{1}(x)=N\left(u_{0}\right)=\frac{-129024 x^{7.5}}{42567525 \sqrt{\pi}}-\frac{x^{7}}{210} \\
& \quad-\frac{x^{12}}{138600}-\frac{x^{17}}{179928000}, \\
& u_{2}(x)= \\
& N\left(u_{0}+u_{1}\right)-N\left(u_{0}\right) \\
&=1.71008^{-3} x^{7.5}+5.95252^{-4} x^{8} \\
&+7.21501^{-6} x^{12}+4.28636^{-6} x^{12.5} \\
&+ 9.09455^{-9} x^{17}+1.31911^{-9} x^{17.5} \\
&-5.97301^{-10} x^{18}+1.20298^{-12} x^{22} \\
&-2.48834^{-12} x^{22.5}-2.96617^{-15} x^{27} \\
&-1.02289^{-15} x^{27.5}-2.69485^{-18} x^{32} \\
&-6.62568^{-28} x^{37},
\end{aligned}
$$

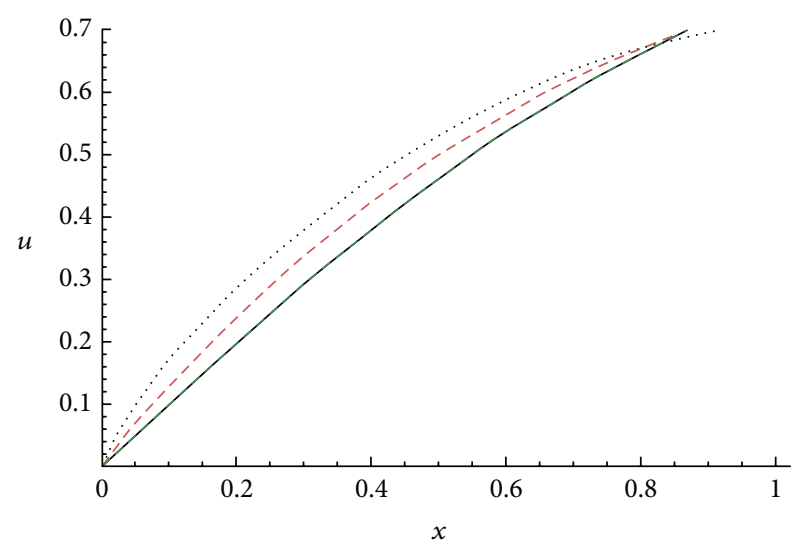

FIGURE 2: Plots of the approximate solution for different values of $\alpha$ and the exact solution for (23).

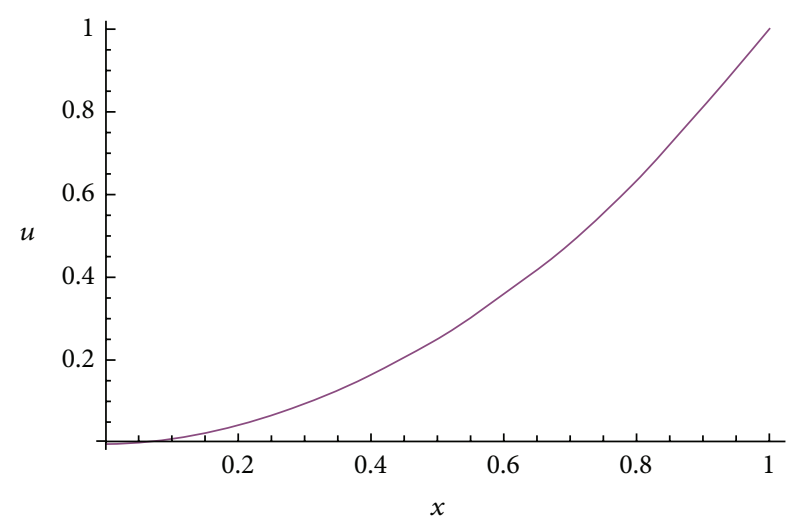

FIgURE 3: Plots of the approximate solution and the exact solution for (27).

and so on. The 4-term approximate solution and the corresponding exact solution for (27) are plotted in Figure 3. It is remarkable to note that the two solutions are almost equal.

Comparing these obtained results with those obtained by new Jacobi operational matrix in $[23,24]$, we can confirm the simplicity and accuracy of the given method.

Example 6. Consider the following fractional order wave equation in 2-dimensional space [14]:

$$
\begin{aligned}
& D_{* t}^{\alpha} u(x, y, t)+c\left(u_{x}+u_{y}\right)=0, \\
& u(x, y, 0)=\sin \left[\pi\left(\frac{x+y}{l}\right)\right], \quad 0<\alpha \leq 1 .
\end{aligned}
$$

The exact solution for this problem when $\alpha=1$ is

$$
u(x, y, t)=\sin \left[\pi\left(\frac{x+y-2 c t}{l}\right)\right] .
$$

The initial value problem (30) is equivalent to the integral equation

$$
u(x, y, t)=\sin \left[\pi\left(\frac{x+y}{l}\right)\right]-I_{t}^{\alpha}\left[c\left(u_{x}+u_{y}\right)\right] .
$$


Let $N(u)=-I_{t}^{\alpha}\left[c\left(u_{x}+u_{y}\right)\right]$. In view of recurrence relation (17), we have the following first approximations:

$$
\begin{gathered}
u_{0}(x, y, t)=\sin \left[\pi\left(\frac{x+y}{l}\right)\right], \\
u_{1}(x, y, t)=\frac{-1}{\Gamma(1+\alpha)}\left(\frac{2 c \pi t^{\alpha}}{l}\right) \cdot \cos \left[\pi\left(\frac{x+y}{l}\right)\right], \\
u_{2}(x, y, t)=\frac{-1}{\Gamma(1+2 \alpha)}\left(\frac{2 c \pi t^{\alpha}}{l}\right)^{2} \cdot \sin \left[\pi\left(\frac{x+y}{l}\right)\right], \\
u_{3}(x, y, t)=\frac{1}{\Gamma(1+3 \alpha)}\left(\frac{2 c \pi t^{\alpha}}{l}\right)^{3} \cdot \cos \left[\pi\left(\frac{x+y}{l}\right)\right],
\end{gathered}
$$

and so on. The $n$-term approximate solution for (30) is

$$
\begin{aligned}
& u(x, y, t)=\sum_{i=0}^{n-1} u_{i} \\
& =\sin \left[\pi\left(\frac{x+y}{l}\right)\right] \\
& \times\left[1-\frac{1}{\Gamma(1+2 \alpha)}\left(\frac{2 c \pi t^{\alpha}}{l}\right)^{2}\right. \\
& +\frac{1}{\Gamma(1+4 \alpha)}\left(\frac{2 c \pi t^{\alpha}}{l}\right)^{4} \\
& \left.-\frac{1}{\Gamma(1+6 \alpha)}\left(\frac{2 c \pi t^{\alpha}}{l}\right)^{6}+\cdots\right] \\
& -\cos \left[\pi\left(\frac{x+y}{l}\right)\right] \\
& \times\left[\frac{1}{\Gamma(1+\alpha)}\left(\frac{2 c \pi t^{\alpha}}{l}\right)\right. \\
& -\frac{1}{\Gamma(1+3 \alpha)}\left(\frac{2 c \pi t^{\alpha}}{l}\right)^{3} \\
& +\frac{1}{\Gamma(1+5 \alpha)}\left(\frac{2 c \pi t^{\alpha}}{l}\right)^{5} \\
& \left.-\frac{1}{\Gamma(1+7 \alpha)}\left(\frac{2 c \pi t^{\alpha}}{l}\right)^{7}+\cdots\right] \text {. }
\end{aligned}
$$

In closed form this gives:

$$
\begin{aligned}
u(x, y, t)= & \sin \left[\pi\left(\frac{x+y}{l}\right)\right] \\
& \cdot \cos \left(\frac{2 c \pi t^{\alpha}}{l}\right) \\
& -\cos \left[\pi\left(\frac{x+y}{l}\right)\right], \\
\sin \left(\frac{2 c \pi t^{\alpha}}{l}\right)= & \sin \left[\pi\left(\frac{x+y-2 c t^{\alpha}}{l}\right)\right]
\end{aligned}
$$

which is the exact solution for the given problem. When $\alpha=$ 1 , the above $n$-term approximate solution for (30) becomes

$$
\begin{aligned}
u(x, y, t)= & \sum_{i=0}^{n-1} u_{i} \\
= & \sin \left[\pi\left(\frac{x+y}{l}\right)\right] \\
& \times\left[1-\frac{1}{2 !}\left(\frac{2 c \pi t}{l}\right)^{2}+\frac{1}{4 !}\left(\frac{2 c \pi t}{l}\right)^{4}\right. \\
& -\cos \left[\pi\left(\frac{x+y}{6 !}\right)\right] \\
& \times\left[\left(\frac{2 c \pi t}{l}\right)^{6}+\cdots\right] \\
& \left.\quad+\frac{1}{5 !}\left(\frac{2 c \pi t}{l}\right)^{5}-\frac{1}{7 !}\left(\frac{2 c \pi t}{l}\right)^{7}+\cdots\right] .
\end{aligned}
$$

In closed form, this gives

$$
\begin{aligned}
& u(x, y, t)= \sin \left[\pi\left(\frac{x+y}{l}\right)\right] \\
& \cdot \cos \left(\frac{2 c \pi t}{l}\right)-\cos \left[\pi\left(\frac{x+y}{l}\right)\right], \\
& \sin \left(\frac{2 c \pi t}{l}\right)=\sin \left[\pi\left(\frac{x+y-2 c t}{l}\right)\right],
\end{aligned}
$$

which is the same result obtained by variational iteration method in [14].

Example 7. Consider the following fractional order heat equation in 2-dimensional space [11]:

$$
\begin{gathered}
D_{* t}^{\beta} u(x, y, t)=\alpha\left(u_{x x}+u_{y y}\right), \\
u(x, y, 0)=c\left[\sin \left(\frac{\pi x}{l}\right)+\sin \left(\frac{\pi y}{l}\right)\right], \quad 0<\beta \leq 1 .
\end{gathered}
$$

The exact solution for this problem when $\beta=1$ is

$$
u(x, y, t)=c e^{-\alpha \pi^{2} t / l^{2}}\left[\sin \left(\frac{\pi x}{l}\right)+\sin \left(\frac{\pi y}{l}\right)\right]
$$

The initial value problem (38) is equivalent to the integral equation

$u(x, y, t)=c\left[\sin \left(\frac{\pi x}{l}\right)+\sin \left(\frac{\pi y}{l}\right)\right]+I_{t}^{\beta}\left[\alpha\left(u_{x x}+u_{y y}\right)\right]$. 
Let $N(u)=I_{t}^{\beta}\left[\alpha\left(u_{x x}+u_{y y}\right)\right]$. In view of recurrence relation (17), we have the following first approximations:

$$
\begin{aligned}
u_{0}(x, y, t)= & c\left[\sin \left(\frac{\pi x}{l}\right)+\sin \left(\frac{\pi y}{l}\right)\right], \\
u_{1}(x, y, t)= & \frac{-c}{\Gamma(1+\beta)}\left(\frac{\alpha \pi^{2} t^{\beta}}{l^{2}}\right) \\
& \times\left[\sin \left(\frac{\pi x}{l}\right)+\sin \left(\frac{\pi y}{l}\right)\right], \\
u_{2}(x, y, t)= & \frac{c}{\Gamma(1+2 \beta)}\left(\frac{\alpha \pi^{2} t^{\beta}}{l^{2}}\right)^{2} \\
& \times\left[\sin \left(\frac{\pi x}{l}\right)+\sin \left(\frac{\pi y}{l}\right)\right], \\
u_{3}(x, y, t)= & \frac{-c}{\Gamma(1+3 \beta)}\left(\frac{\alpha \pi^{2} t^{\beta}}{l^{2}}\right)^{3} \\
& \times\left[\sin \left(\frac{\pi x}{l}\right)+\sin \left(\frac{\pi y}{l}\right)\right],
\end{aligned}
$$

and so on. The $n$-term approximate solution for (38) is

$$
\begin{aligned}
u(x, y, t)= & \sum_{i=0}^{n-1} u_{i} \\
= & c\left[\sin \left(\frac{\pi x}{l}\right)+\sin \left(\frac{\pi y}{l}\right)\right] \\
& \times\left[1-\frac{1}{\Gamma(1+\beta)}\left(\frac{\alpha \pi^{2} t^{\beta}}{l^{2}}\right)\right. \\
& +\frac{1}{\Gamma(1+2 \beta)}\left(\frac{\alpha \pi^{2} t^{\beta}}{l^{2}}\right)^{2} \\
& \left.-\frac{1}{\Gamma(1+3 \beta)}\left(\frac{\alpha \pi^{2} t^{\beta}}{l^{2}}\right)^{3}+\cdots\right] .
\end{aligned}
$$

When $\beta=1$, The $n$-term approximate solution for (38) becomes

$$
\begin{aligned}
u(x, y, t)= & \sum_{i=0}^{n-1} u_{i} \\
= & c\left[\sin \left(\frac{\pi x}{l}\right)+\sin \left(\frac{\pi y}{l}\right)\right] \\
& \times\left[1-\frac{1}{1 !}\left(\frac{\alpha \pi^{2} t}{l^{2}}\right)+\frac{1}{2 !}\left(\frac{\alpha \pi^{2} t}{l^{2}}\right)^{2}\right. \\
& \left.\quad-\frac{1}{3 !}\left(\frac{\alpha \pi^{2} t}{l^{2}}\right)^{3}+\cdots\right] .
\end{aligned}
$$

In closed form, this gives

$$
u(x, y, t)=c e^{-\alpha \pi^{2} t / l^{2}}\left[\sin \left(\frac{\pi x}{l}\right)+\sin \left(\frac{\pi y}{l}\right)\right]
$$

which is the exact solution for the given problem.
The obtained results in this example are the same as these obtained in [11] by the homotopy perturbation method, in case $\beta=1$, but with the simplicity of the given method.

Example 8. In this last example, we consider the following fractional order nonlinear wave equation [25]:

$$
\begin{aligned}
& D_{* x}^{\alpha} u(x, t)-u u_{t t}=\frac{x^{2-\alpha}}{\Gamma(3-\alpha)}-\frac{\left(x^{2}+t^{2}\right)}{2}, \\
& u(0, t)=\frac{t^{2}}{2}, \quad u_{x}(0, t)=0, \quad 1<\alpha \leq 2 .
\end{aligned}
$$

The exact solution for this problem when $\alpha=2$ is $u(x, t)=$ $(1 / 2)\left(x^{2}+t^{2}\right)$ where $0 \leq x, t \leq 1$.

The initial value problem (45) is equivalent to the integral equation

$$
u(x, t)=\frac{1}{2}\left(x^{2}+t^{2}\right)-\frac{x^{2+\alpha}}{\Gamma(3+\alpha)}-\frac{t^{2} x^{\alpha}}{2 \Gamma(1+\alpha)}+I_{x}^{\alpha}\left[u u_{t t}\right] .
$$

Let $N(u)=I_{x}^{\alpha}\left[u u_{t t}\right]$. In view of recurrence relation (8), we have

$$
\begin{aligned}
u_{0}(x, t)= & \frac{1}{2}\left(x^{2}+t^{2}\right) \\
& -\frac{x^{2+\alpha}}{\Gamma(3+\alpha)}-\frac{t^{2} x^{\alpha}}{2 \Gamma(1+\alpha)}, \\
u_{1}(x, t)= & \frac{x^{2+\alpha}}{\Gamma(3+\alpha)}-\frac{x^{2+2 \alpha}}{\Gamma(3+2 \alpha)} \\
& -\frac{\Gamma(3+\alpha) x^{2+2 \alpha}}{2 \Gamma(1+\alpha) \Gamma(3+2 \alpha)} \\
& +\frac{\Gamma(3+2 \alpha) x^{2+3 \alpha}}{\Gamma(1+\alpha) \Gamma(3+\alpha) \Gamma(3+3 \alpha)} \\
& +t^{2}\left(\frac{x^{\alpha}}{2 \Gamma(1+\alpha)}-\frac{x^{2 \alpha}}{\Gamma(1+2 \alpha)}\right. \\
& \left.+\frac{\Gamma(1+2 \alpha) x^{3 \alpha}}{2 \Gamma(1+\alpha)^{2} \Gamma(1+3 \alpha)}\right),
\end{aligned}
$$

and so on. The 3-term approximate solution and the corresponding exact solution for (45) are plotted in Figure 4(a), in case $t=1 / 2$, for $\alpha=1.8,1.9,2$., in Figure 4(b), in case $t=1$, for $\alpha=1.8,1.9,2$, and in Figure 4(c), in case $\alpha=2$. It is remarkable to note that in the first two figures all the solutions are almost equal.

Comparing these results with those obtained by the modification homotopy perturbation method in [25], we can confirm the accuracy and simplicity of the given method.

\section{Conclusion}

In this paper, the new iterative method with suitable algorithm is successfully used to solve linear and nonlinear 


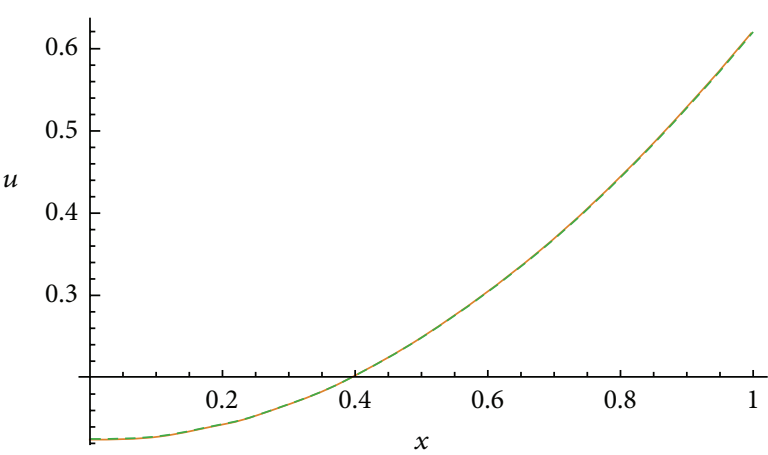

(a)

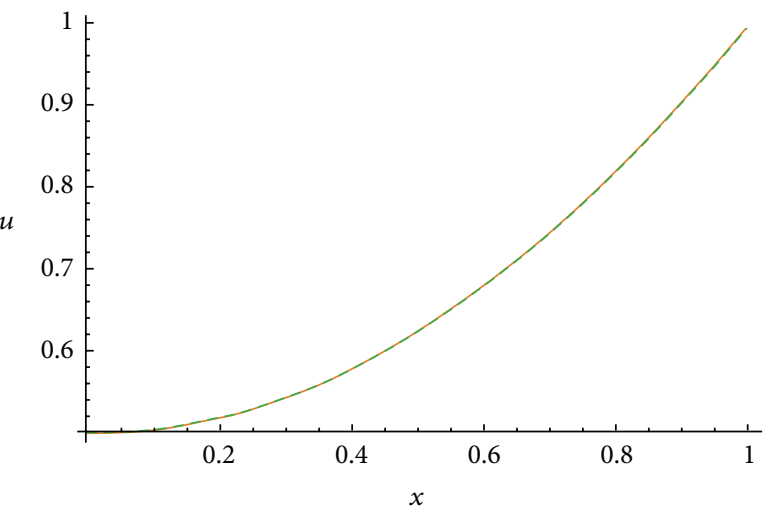

(b)

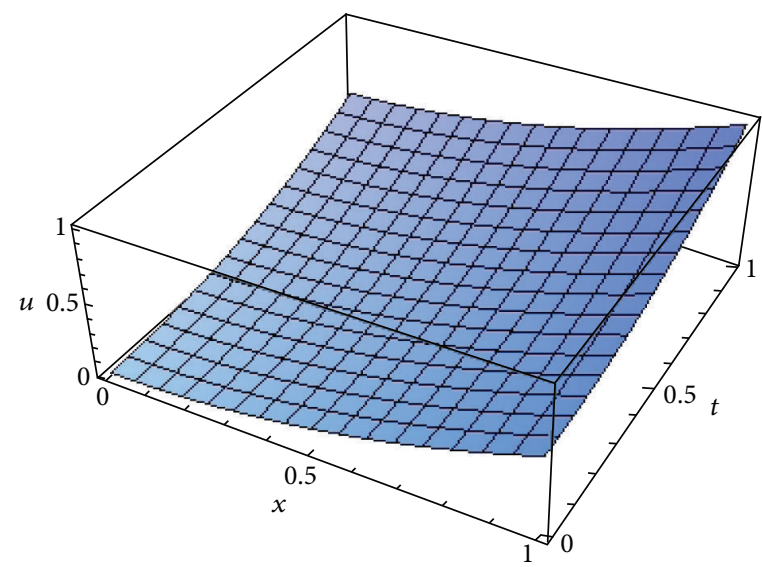

(c)

Figure 4: (a) Plots of the approximate solution for different values of $\alpha$ and the exact solution, in case $t=1 / 2$; for (45). (b) Plots of the approximate solution for different values of $\alpha$ and the exact solution, in case $t=1$; for (45). (c) Plots of the approximate solution, in case $\alpha=2$ for (45).

ordinary and partial differential equations with fractional order. It is clear that the computations are easy and the solutions agree well with the corresponding exact solutions and more accurate than the solutions obtained by other methods. Moreover, the accuracy is high with little computed terms of the solution which confirm that this method with the given algorithm is a powerful method for handling fractional differential equations.

\section{References}

[1] K. Miller and B. Ross, An Introduction to the Fractional Calculus and Fractional Diffrential Equations, John Wiley \& Sons, New York, NY, USA, 1993.

[2] K. B. Oldham and J. Spanier, The Fractional Calculus, Academic Press, London, UK, 1974.

[3] I. Podlubny, Fractional Differential Equations, vol. 198, Academic Press, San Diego, Calif, USA, 1999.

[4] M. Amairi, M. Aoun, S. Najar, and M. N. Abdelkrim, "A constant enclosure method for validating existence and uniqueness of the solution of an initial value problem for a fractional differential equation," Applied Mathematics and Computation, vol. 217, no. 5, pp. 2162-2168, 2010.
[5] J. Deng and L. Ma, "Existence and uniqueness of solutions of initial value problems for nonlinear fractional differential equations," Applied Mathematics Letters, vol. 23, no. 6, pp. 676$680,2010$.

[6] E. Girejko, D. Mozyrska, and M. Wyrwas, "A sufficient condition of viability for fractional differential equations with the Caputo derivative," Journal of Mathematical Analysis and Applications, vol. 381, no. 1, pp. 146-154, 2011.

[7] S. S. Ray and R. K. Bera, "Solution of an extraordinary differential equation by Adomian decomposition method," Journal of Applied Mathematics, no. 4, pp. 331-338, 2004.

[8] M. Dehghan, J. Manafian, and A. Saadatmandi, "Solving nonlinear fractional partial differential equations using the homotopy analysis method," Numerical Methods for Partial Differential Equations, vol. 26, no. 2, pp. 448-479, 2010.

[9] I. Hashim, O. Abdulaziz, and S. Momani, "Homotopy analysis method for fractional IVPs," Communications in Nonlinear Science and Numerical Simulation, vol. 14, no. 3, pp. 674-684, 2009.

[10] Z. Odibat, S. Momani, and H. Xu, "A reliable algorithm of homotopy analysis method for solving nonlinear fractional differential equations," Applied Mathematical Modelling, vol. 34, no. 3, pp. 593-600, 2010. 
[11] A. A. Hemeda, "Homotopy perturbation method for solving partial differential equations of fractional order," International Journal of Mathematical Analysis, vol. 6, no. 49-52, pp. 24312448, 2012.

[12] A. A. Hemeda, "Homotopy perturbation method for solving systems of nonlinear coupled equations," Applied Mathematical Sciences, vol. 6, no. 93-96, pp. 4787-4800, 2012.

[13] S. Esmaeili, M. Shamsi, and Y. Luchko, "Numerical solution of fractional differential equations with a collocation method based on Müntz polynomials," Computers \& Mathematics with Applications, vol. 62, no. 3, pp. 918-929, 2011.

[14] A. A. Hemeda, "Variational iteration method for solving wave equation," Computers \& Mathematics with Applications, vol. 56, no. 8, pp. 1948-1953, 2008.

[15] A. A. Hemeda, "Variational iteration method for solving nonlinear partial differential equations," Chaos, Solitons and Fractals, vol. 39, no. 3, pp. 1297-1303, 2009.

[16] A. A. Hemeda, "Variational iteration method for solving nonlinear coupled equations in 2-dimensional space in fluid mechanics," International Journal of Contemporary Mathematical Sciences, vol. 7, no. 37-40, pp. 1839-1852, 2012.

[17] M. G. Sakar, F. Erdogan, and A. Yıldırım, "Variational iteration method for the time-fractional Fornberg-Whitham equation," Computers \& Mathematics with Applications, vol. 63, no. 9, pp. 1382-1388, 2012.

[18] E. H. Doha, A. H. Bhrawy, and S. S. Ezz-Eldien, "Efficient Chebyshev spectral methods for solving multi-term fractional orders differential equations," Applied Mathematical Modelling, vol. 35, no. 12, pp. 5662-5672, 2011.

[19] E. H. Doha, A. H. Bhrawy, and S. S. Ezz-Eldien, "A Chebyshev spectral method based on operational matrix for initial and boundary value problems of fractional order," Computers \& Mathematics with Applications, vol. 62, no. 5, pp. 2364-2373, 2011.

[20] V. Daftardar-Gejji and H. Jafari, "An iterative method for solving nonlinear functional equations," Journal of Mathematical Analysis and Applications, vol. 316, no. 2, pp. 753-763, 2006.

[21] A. A. Hemeda, "New iterative method: application to nthorder integro-differential equations," International Mathematical Forum, vol. 7, no. 47, pp. 2317-2332, 2012.

[22] A. A. Hemeda, "Formulation and solution of $n$ th-order derivative fuzzy integrodifferential equation using new iterative method with a reliable algorithm," Journal of Applied Mathematics, vol. 2012, Article ID 325473, 17 pages, 2012.

[23] A. Saadatmandi and M. Dehghan, "A new operational matrix for solving fractional-order differential equations," Computers \& Mathematics with Applications, vol. 59, no. 3, pp. 1326-1336, 2010.

[24] E. H. Doha, A. H. Bhrawy, and S. S. Ezz-Eldien, "A new Jacobi operational matrix: an application for solving fractional differential equations," Applied Mathematical Modelling, vol. 36, no. 10, pp. 4931-4943, 2012.

[25] B. Ghazanfari, A. G. Ghazanfari, and M. Fuladvand, "Modification of the homotopy perturbation method for numerical solution of nonlinear wave and system of nonlinear wave equations," The Journal of Mathematics and Computer Science, vol. 3, no. 2, pp. 212-224, 2011.

[26] M. Caputo, "Linear methods of dissipation whose Q is almost frequency independent, part II," Journal of the Royal Society of Medicine, vol. 13, pp. 529-539, 1967.

[27] Y. Cherruault, “Convergence of Adomian's method," Kybernetes, vol. 18, no. 2, pp. 31-38, 1989.
[28] A. J. Jerri, Introduction to Integral Equations with Applications, Wiley-Interscience, New York, NY, USA, 2nd edition, 1999.

[29] S. Bhalekar and V. Daftardar-Gejji, "Convergence of the new iterative method," International Journal of Differential Equations, vol. 2011, Article ID 989065, 10 pages, 2011. 


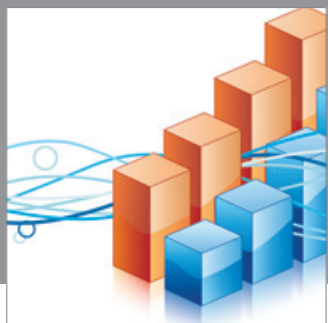

Advances in

Operations Research

mansans

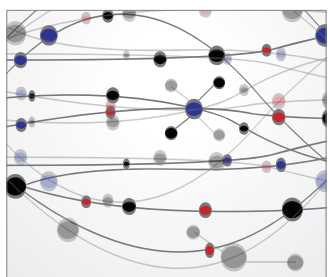

The Scientific World Journal
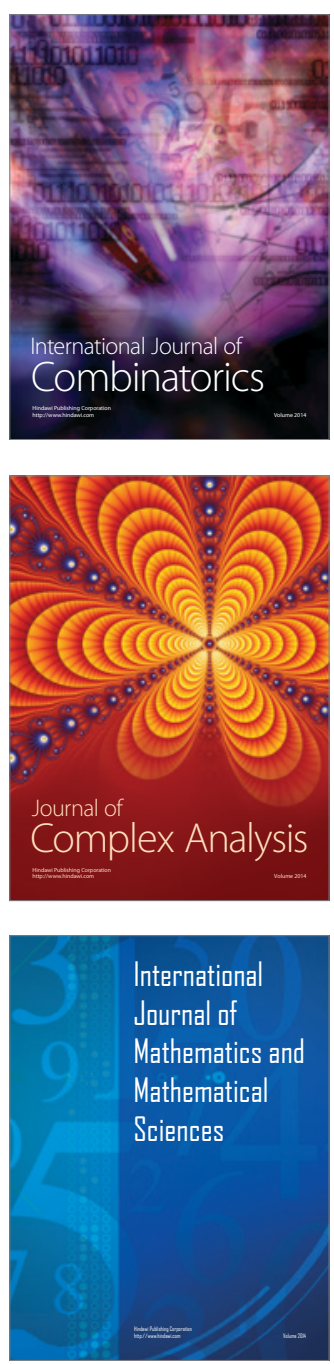
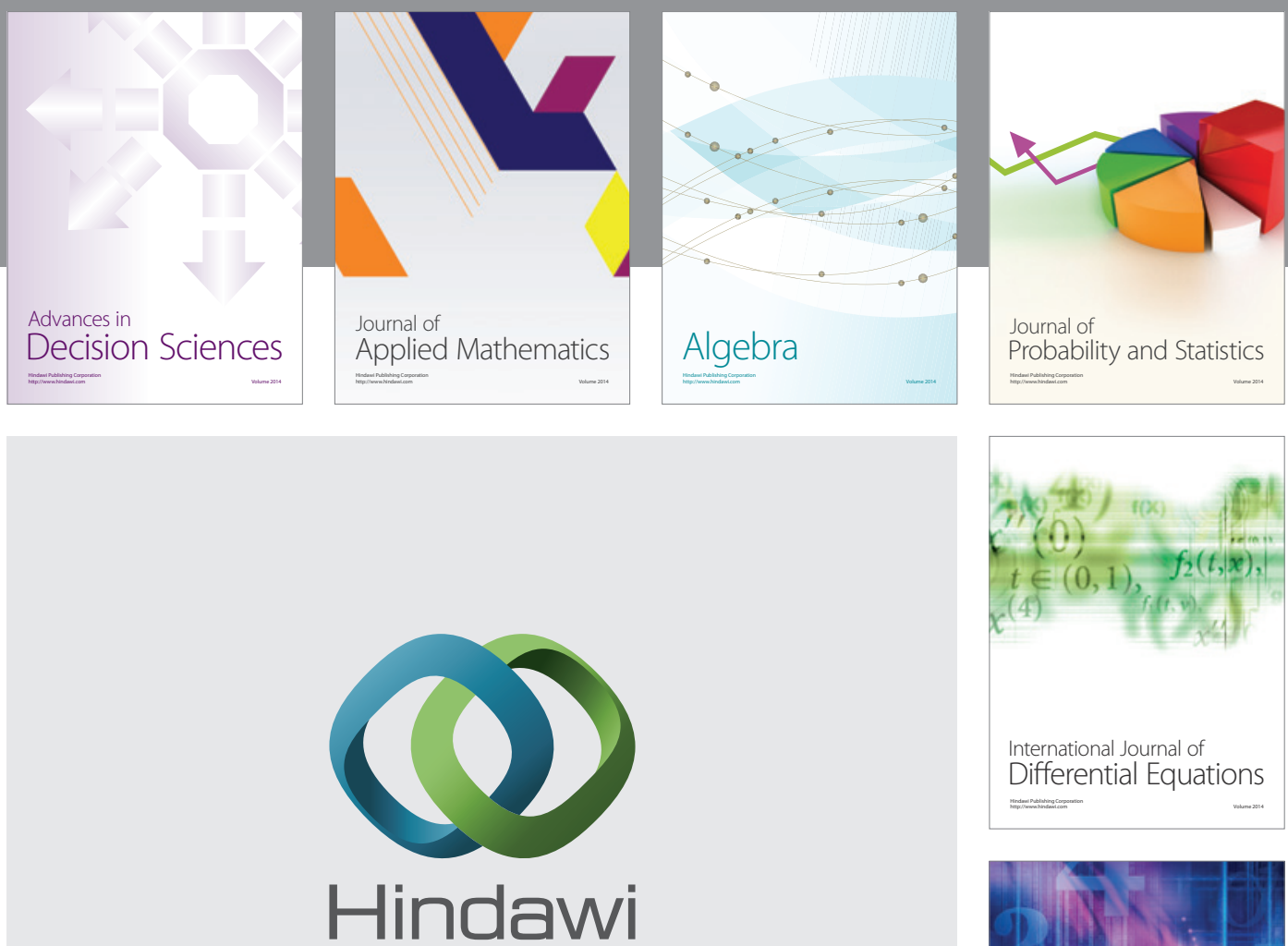

Submit your manuscripts at http://www.hindawi.com
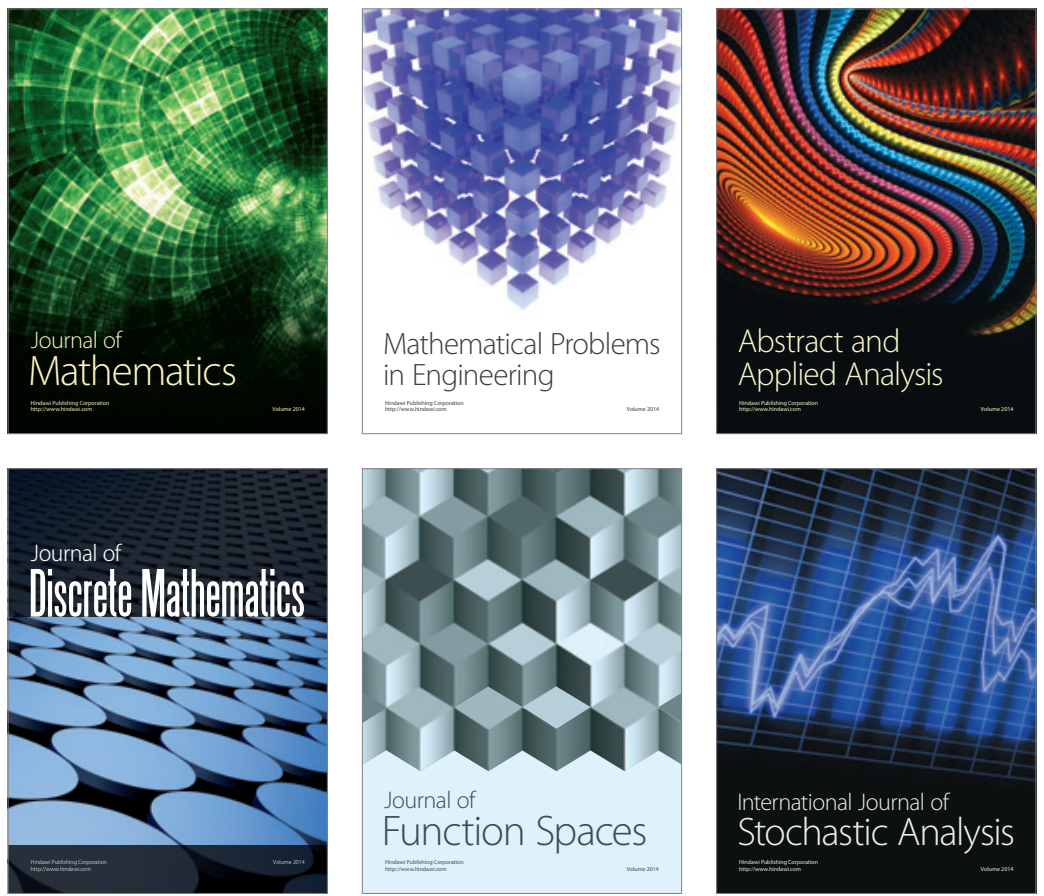

Journal of

Function Spaces

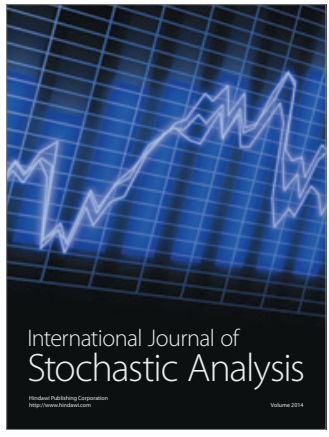

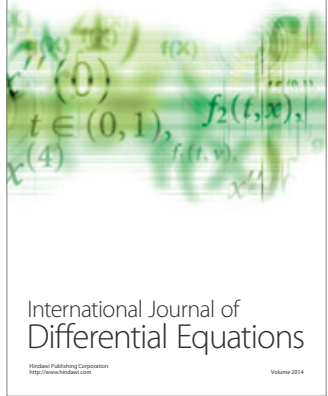
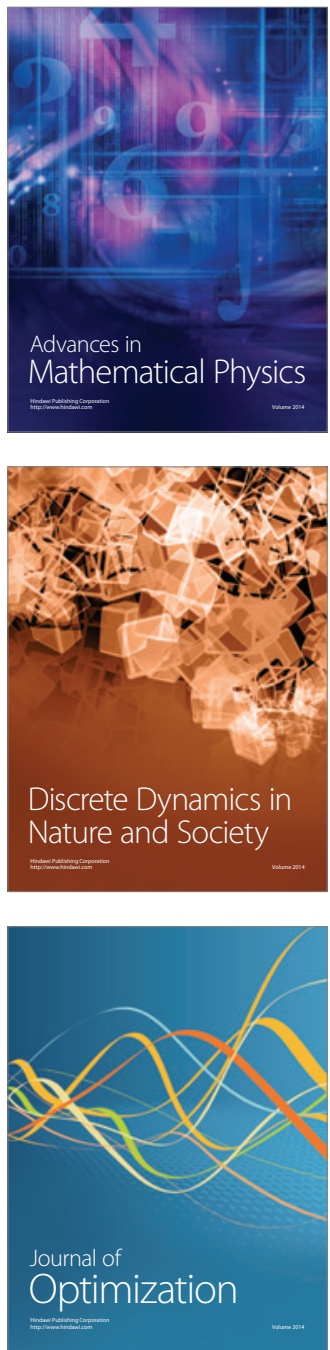